\title{
BRUNO BLECKMANN
}

\section{Römische Politik im Ersten Punischen Krieg ${ }^{1}$}

\section{Zur Bedeutung des Ersten Punischen Kriegs in der inneren Geschichte der Republik}

Der Erste Punische Krieg wird schon im Geschichtswerk des Polybios als ein Ereigniszusammenhang beschrieben, in dem die Römer durch beharrlichen und geschlossenen Einsatz die zunächst auf gleicher Augenhöhe stehende Großmacht Karthago in einem jahrelangen Ringen niederzwingen. Vielleicht hat auch der Zeitgenosse Naevius in seinem der Darstellung des Krieges gewidmeten Epos die beispiellose kollektive Kraftanstrengung und Bewährungsprobe der Republik hervorgehoben. Exemplarische Gestalten wie A. Atilius Calatinus, der im Cato des Cicero gerühmt wird, Q. Caecilius Metellus oder vor allem der Märtyrer für römische Werte M. Atilius Regulus, dessen Legende bereits im Geschichtswerk des Sempronius Tuditanus mit allen bekannten Details versehen war, gaben in der kollektiven Erinnerung dem Ersten Punischen Krieg eine Aura der Erfüllung altrepublikanischer Werte und Harmonie. Der Erste Punische Krieg scheint damit am ehesten zu dem idealisierenden Bild zu passen, das Sallust für die Innenpolitik bis zum Ende der Punischen Kriege entworfen hat:

Vor der Zerstörung Karthagos trieben das römische Volk und der Senat friedlich und besonnen miteinander Politik; es gab unter den Bürgern keinen Streit, weder um Geltung noch um Herrschaft: Furcht vor dem Feind hielt die Bürgerschaft bei ihren guten Eigenschaften. ${ }^{2}$

Nicht ganz unbeeinflusst von diesen idealisierenden, vom scharfen Kontrast zu den Verhältnissen ab der Zeit der Gracchen bestimmten Verklärungen einer angeblich intakten und störungsfreien Zeit charakterisiert Jochen Bleicken die Zeit von der Mitte des vierten Jahrhunderts bis gegen Ende des dritten Jahrhunderts vor Christus als ,eine Zeit verhältnismäßiger Ruhe und Statik“" und rechnet Staveley die gesamte Zeit vor dem Ende des Hannibalkrieges zu einem ,golden age of stable and ordered government. “3

Verglichen mit der Dramatik der Krise der Späten Republik ab den Gracchen, vor allem aber ab den Bürgerkriegen Sullas oder Caesars, mag das dritte Jahrhundert in der Tat als eine Phase relativer innenpolitischer Ruhe erscheinen. Konflikte innerhalb der führenden Schicht, der patrizisch-plebejischen Nobilität, kann man zunächst als selbstverständlichen Bestandteil der politischen Kultur dieser Epoche beschreiben, die nicht an sich das Bild relativer Eintracht modifizieren. Denn die Nobilität, die im dritten Jahrhundert noch nicht

\footnotetext{
${ }^{1}$ In der Hauptsache sind hier in vergröbernder Form die Forschungsergebnisse meiner Monografie von 2002 zusammengefasst; vgl. Bleckmann (2002). Für differenzierende Analysen zur Geschichte der Nobilität der Mittleren Republik ist zu verweisen auf Beck (2005); Hölkeskamp (1987); Hölkeskamp (2004); Hölkeskamp (2006).

2 Sallust, $B J$ 41.2.

3 Calatinus: Cicero, Cato maior 61; vgl. Beck (2005: 230). Zu Regulus: Bleckmann (1998). „Ruhe und Statik“: Bleicken (1975: 371 f.); Staveley (1989: 444).
} 
so eng abgeschlossen war wie in späterer Zeit, sondern etwa noch neuen Eliten aus Italien offenstand, hatte zwar ein gemeinsames Ethos und gemeinsame Normen und Werte und war durch den mos maiorum solidarisch miteinander verbunden. Aber zur gemeinsamen Wertewelt gehörte gerade auch das kompetitive Element. ${ }^{4}$ Nicht von ungefähr gilt einer der bekanntesten Texte, der das kompetitive aristokratische Ethos der Mittleren Republik verdeutlicht, die laudatio funebris für L. Caecilius Metellus, dem Konsul des Jahres 251 und 247 vor Christus, gerade einer Figur aus der Epoche des Ersten Punischen Krieges. In dieser Leichenrede rühmte Q. Metellus seinen Vater in einer Serie von Superlativen und hob die einzelnen in der aristokratischen Kompetition erreichten und von allen anerkannten Bestmarken hervor:

Q. Metellus hat in der Lobrede, die er bei der letzten Ehrung seines Vaters L. Metellus hielt, der Oberpriester, zweimal Konsul, Diktator, Befehlshaber der Reiterei und einer der zur Verteilung von Land erwählten Fünfzehnmänner war und der nach dem ersten Punischen Krieg erstmals Elefanten im Triumph aufführte, schriftlich überliefert, sein Vater habe die zehn höchsten und besten Vorzüge, deren Erlangung die politisch Klugen ihr Leben widmeten, in sich vereinigt: sein Wunsch sei gewesen, der erste Krieger, der beste Redner, der tapferste Feldherr zu sein, die größten Taten unter eigenem Kommando durchzuführen, die höchste Ehrenstelle innezuhaben, von größter Klugheit zu sein, als ranghöchster Senator zu gelten, ein großes Vermögen auf gute Weise zu erwerben, viele Kinder zu hinterlassen und der Berühmteste im Staat zu sein. ${ }^{5}$

Wer der Nobilität angehörte, von dem wurde geradezu erwartet, dass er an dem Sich-gegenseitigen-Messen und am Wettbewerb um militärischen Ruhm, um politischen Einfluss, um Reichtum und Prestige teilnahm. Dieser Wettbewerb wurde dabei nicht als Kampf aller gegen aller ausgetragen, sondern durch Bündnisse und Wahlabsprachen, durch ein Netzwerk von Beziehungen zum gegenseitigen Vorteil und durch die Mobilisierung nicht nur der eigenen Klientel, sondern auch derjenigen der politischen Freunde flankiert. Die Entscheidungen im Senat und die Tagespolitik auf dem Forum war daher geradezu zwangsläufig von ständigen Konflikten begleitet; und römische Politik konnte gar nicht anders durchgeführt werden als im Intrigenspiel und in ständigen Auseinandersetzungen um Macht und Einfluss.

Die Parteiungen, die dabei zusammenarbeiteten, fluktuierten beständig in ihrer Zusammensetzung. Die Annahme kohärenter durch Heiratsverbindungen zusammengehaltener dauerhafter Gruppierungen von Familien, wie den angeblich zusammenwirkenden Claudiern und Atiliern oder den mit den Aemiliern verbunden Fabiern, wie sie von der älteren prosopografischen Forschung vertreten wurde, hat sich bei kritischer Betrachtung des Quellenmaterials als illusionär erwiesen. Vollends ausgeschlossen ist, dass diese festen Adelsgruppen dann auch noch mit programmatischen Positionen in Innen- und Außenpolitik verbunden waren, etwa einem konservativen Kurs der Nordexpansion und einem radikalpopulistischen Kurs der Expansion in den Süden. Den einzigen, etwas festeren Typus politischer Zusammenarbeit, den man gerade für die Epoche des Ersten Punischen Krieges feststellen kann, ist diejenige zwischen unmittelbaren Verwandten. In den Fasten lassen sich mehrfach Brüder oder auch Vettern ersten Grades nachweisen, etwa die Brüder M'. Otacilius Crassus, cos. 263, und T. Otacilius Crassus, cos. 261, die Brüder Q. Lutatius Catulus, cos. 242, und C. Lutatius Cerco, cos. 241, die Vettern beziehungsweise Brüder

\footnotetext{
4 Vgl. hierzu die zahlreichen Arbeiten Hölkeskamps (s. Fußnote 1).

5 Plinius, $N H 7.139 \mathrm{f}$.
} 
A. Atilius Caiatinus, cos. 258, und C. Atilius Regulus, cos. 257, Vetter seines Vorgängers, und M. Atilius Regulus, cos. suff. 256 und Bruder des Konsuls von 256. Erklärbar sind diese Familiencluster dadurch, dass ein Amtsträger seine Macht und sein Prestige durch die Wahl eines nahen Verwandten deutlich machen konnte, aber auch durch das Bestreben, eigene Maßnahmen und politische Entscheidungen über das eigene Amtsjahr hinaus bestehen lassen zu können. Letzteres wird besonders im Falle des Prokonsuls Q. Lutatius Catulus deutlich, dessen in Rom hochumstrittene Friedensvereinbarungen durch das geschickte Agieren seines Bruders durchgesetzt wurden. ${ }^{6}$

Innenpolitische Konflikte gehörten also als Folge inneraristokratischen Wettbewerbs zum System, nach dem Politik und Gesellschaft in der Republik funktionierten. Nuancen sind aber in der Art und Weise möglich, in der die Balance zwischen dem kompetitiven Element und dem aristokratischen Grundkonsens beschrieben wird, und zwar gerade für den Ersten Punischen Krieg. Angesichts gravierender Veränderungen im Institutionengefüge und der erst im Verlauf des dritten Jahrhunderts erfolgenden Verregelung, die mit der Festlegung des cursus honorum durch die lex Villia Annalis einen vorläufigen Abschluss fand, sind auf jeden Fall die ersten Jahrzehnte der Mittleren Republik von einer Dynamik gezeichnet, die durchaus mit den gravierenden außenpolitischen Veränderungen mithalten kann.

Dabei sind die inneren Konflikte in dieser Umbruchzeit möglicherweise schwerer gewesen, als landläufig angenommen. Gewiss erscheint es übertrieben, wenn man die Auseinandersetzungen dieser Zeit in eine ununterbrochene Entwicklungslinie stellt, die von den Ständekämpfen zu den Konflikten der ausgehenden römischen Republik reicht. Aber ein Teil der inneren Konflikte ist auch schon im dritten Jahrhundert mit einer Erbitterung und Kompromisslosigkeit durchgeführt worden, die durchaus mit der späteren Epoche der Bürgerkriege vergleichbar ist. Sie hat nur deshalb viel geringere Folgen, weil die Machtmittel der Kontrahenten in dieser Zeit noch nicht genügten, um aus dem eigenen Vermögen Armeen zu bezahlen. Ein Mindestmaß an Konsens wurde in dieser Zeit dabei schon durch den einfachen Umstand erzwungen, dass die einzelnen Nobiles nur dann in größerem Maßstab Prestige und Macht erwerben konnten, wenn sie auf die staatlichen Organisationsleistungen zurückgriffen. Die wenigen Wochen, in denen ein Aristokrat als römischer Konsul über das Kommando der für eine Sommerkampagne aufgebotenen Militärmacht verfügte, wurden aber so genutzt, dass der eigene Erfolg im Vordergrund stand, bevor im nächsten Jahr ein anderer die gleiche Chance erhielt. Dementsprechend dominierte nicht die pietätvolle Absprache mit dem Senat, sondern das oft ohne Rücksicht auf Verluste betriebene Bestreben, in kürzester Zeit den größtmöglichen persönlichen Ruhm zu sammeln.

Diese Eigenart einer kurzatmigen, vom Prestigestreben einzelner Individuen vorangetriebenen römischen Kriegführung wird jedenfalls dann deutlich, wenn man sich von dem von Polybios gebotenen Bild des heroisch ringenden römischen Kollektivs löst und stärker die Perspektive des Dio-Zonaras einnimmt, in der der Erste Punische Krieg vor allem als eine Serie ehrgeiziger Kampagnen einzelner Generäle aufgefasst wird - ein Bild, das in dem von Polybios benutzten annalistischen Quellenmaterial gelegentlich durchaus auch

\footnotetext{
6 Illusionäre Heiratsverbindungen: vgl. vor allem Lippold (1963); Càssola (1962) und (für das Zusammenwirken zwischen Aemiliern und Fabiern) Münzer (1920); überblick über die diversen Ableger der Faktionentheorie bei Hölkeskamp (2001). Zu Verwandten: vgl. Beck (2005: 129 f.).
} 
immer wieder aufscheint. ${ }^{7}$ Komplettiert wird diese wenig gefällige Skizze innernobilitärer Beziehungen im Ersten Punischen Krieg durch Nachrichten von Kämpfen um die Zuweisung von Kommanden, von Möglichkeiten zur lukrativen Kriegführung und von Versuchen, Karrieren durch Prozesse zu behindern. Deutlich wird schließlich, dass militärische Leistungsfähigkeit und imperiale Expansion nicht trotz dieser innenpolitischen Verhältnisse, sondern gerade wegen dieser Rivalitäten möglich waren, die ganz entscheidend den überaus kühnen und aggressiven Kriegskurs der einzelnen im höchsten Maße ehrgeizigen Konsuln erklären. Auch hier lassen sich Analogien zur Späten Republik herstellen, in der die imperiale Expansion mit der Steigerung innerer Konflikte Schritt hielt.

\section{Der Auftakt des Ersten Punischen Kriegs}

Dieses Bild der inneraristokratischen Beziehungen in der Zeit des Ersten Punischen Krieges mag in einigen Punkten absichtsvoll überzeichnet worden sein, lässt sich aber immerhin durch die Interpretation einer ganzen Reihe entscheidender Episoden des Krieges stützen, dessen Verlauf und Wendungen teilweise eben nicht durch kriegerische Entscheidungen, sondern durch die Dynamik der Innenpolitik und der Rivalität zwischen Nobiles erklärbar sind. Das beginnt bereits mit der Entscheidung, 264 das Hilfeersuchen der Mamertiner zu akzeptieren und ein konsularisches Heer nach Messana gehen zu lassen. Diese Entscheidung wurde, wie die Darstellung des Polybios erkennen lässt, in einer heftigen Auseinandersetzung zwischen dem Senat (oder besser der Mehrheit des Senats) und den Konsuln gefällt. Unmittelbare Sicherheitsinteressen Roms, die vielleicht eine Kriegsentscheidung im Konsens herbeigeführt hatten, waren bei der Entscheidung für den Kriegseintritt nicht tangiert. Denn die Karthager waren um diese Zeit keineswegs in dem von Polybios dargestellten Umfang bedrohlich. Ferner war die bevorstehende Unterwerfung der kampanischen Mamertiner durch Syrakus eine sizilische Angelegenheit und hatte keine unmittelbaren Auswirkungen auf die römische Herrschaft in Italien. In der Hauptsache ist die Entscheidung für den Kriegseintritt allein dem Ehrgeiz des Konsuls Appius Claudius zuzuweisen, dem aufgrund schon lang bestehender Beziehungen zwischen oskischen Söldnergruppen und Kampanern die reichen Städte Siziliens mit ihren lukrativen Beutemöglichkeiten bekannt waren. Im Appell der Kampaner von Messana sah er eine ideale Gelegenheit gekommen, in seinem Konsulat militärisches Prestige und materielle Mittel zu gewinnen, die mit der seinem Kollegen Fulvius gebotenen Chance gleichkamen, die Fortführung der Eroberung von Volsinii zu betreiben. ${ }^{8}$

Die Widerstände, mit denen Claudius sich im römischen Senat konfrontiert sah, waren kaum durch philanthropische Skrupel bedingt, aufgrund derer man den Mamertinern als Feinden der griechischen Städte keine Hilfe angedeihen lassen konnte, noch durch die

\footnotetext{
7 Die Qualität der relativ ausführlichen Erzählung des Cassius Dio (meist in der Zusammenfassung des Zonaras) wird kontrovers diskutiert. Dass sie von höherem Wert ist als die (nur sehr unvollständig zu rekonstruierende) livianische Tradition, lässt sich an Einzelfällen nachweisen, etwa in der Darstellung der missglückten Fahrt des L. Cornelius Scipio 259 oder des Friedens von 241, wo bei Livius bereits Sardinien abgetreten worden ist.

8 Die Entscheidung von 264: vgl. auch Bleckmann (2002: 63-77) mit einer eingehenden Kritik des detaillierten Berichts bei Polybius 1.10-11. Karthager keineswegs bedrohlich: vgl. hierzu die Kritik von Heuss $\left({ }^{3} 1970\right.$ : 26 f.). Oskische Söldnergruppen und Kampaner: Bleckmann (1999). Zur Bedeutung des Krieges gegen Volsinii: Bleckmann (2002: 68-70). Dieser Krieg wurde erst durch Fulvius Flaccus 264 und nicht durch Q. Fabius Gurges 265 abgeschlossen, vgl. CIL 6.40895-96; FTr S. 547 Degrassi; Festus s.v. picta, S. 228 Lindsay; Properz 4.2.3-4.
} 
Befürchtung, dass von der strategisch richtigen Nordexpansion abgewichen wurde. Vielmehr ging es einer Senatsmehrheit darum, einem Claudier die Möglichkeit zu verwehren, durch exzessive Beute in der aristokratischen Kompetition entscheidende Vorteile zu gewinnen. Aus diesem Grunde bediente sich Claudius - gegen den Widerstand des Senats, aber anscheinend im Einverständnis mit seinem Kollegen Fulvius - der Volksversammlung, die er dazu veranlasste, die Unterstützung der Mamertiner zu beschließen und ihm das Kommando für diesen Feldzug zu verleihen. ${ }^{9}$

Über die Erfolge des Claudius in dieser ersten Kampagne sind sich die Quellen uneins. Das hängt damit zusammen, dass die römische Historiografie von parteiischen Familientraditionen beeinflusst ist, die die Erfolge der eigenen gens verherrlichten und die rivalisierender gentes zu schmälern suchten. Die in Wirklichkeit anscheinend weder überragenden noch besonders verheerenden Ergebnisse des Feldzugs wurden vom Senat, dessen Mehrheitswillen von Claudius ignoriert worden war, demonstrativ nicht mit einem Triumph belohnt. Nur die claudische Familientradition weiß von einem Triumph des Konsuls von 264, der wohl nicht frei erfunden worden ist, sondern als eine private, ohne Senatsabschluss durchgeführte Prestigedemonstration stattgefunden haben dürfte. ${ }^{10}$

Die Kampagne des Jahres 263 konnte auf den von Claudius geschaffenen Voraussetzungen aufbauen. Diesmal erhielten beide Konsuln M'. Valerius und M'. Otacilius das reiche Beute versprechende Aktionsfeld Sizilien. Im Kampf gegen Hieron von Syrakus gingen beide konsularische Heere dabei nur teilweise gemeinsam vor, teilweise marschierten sie getrennt. Ein solches Vorgehen hing mit den Schwierigkeiten der Versorgung einer großen Truppe und der Möglichkeit zusammen, auf diese Weise in den kleineren Städten Ostsiziliens besonders rasch Beute einsammeln zu könnnen. Allerdings fehlte auch hier wieder nicht ein Element inneraristokratischer Rivalität. Denn M'. Valerius scheint alles daran gesetzt zu haben, sich durch die getrennte Kriegführung als der allein herausragende Feldherr hervorzuheben. Aus der Beute von Catana ließ er allein in seinem Namen eine Sonnenuhr auf dem Forum Romanum installieren. ${ }^{11}$ Vor allem aber nahm er für sich in Anspruch, Messana aus dem karthagisch-syrakusanischen Belagerungsring befreit zu haben, weshalb er den Beinamen Messala erhielt. Die Kampagne von 263 war, anders als Polybios suggeriert, mit dem schließlich gegen Hieron II. durchgesetzten Friedensvertrag nicht beendet. Weil die Ambitionen der Konsuln mit dem Erfolg gegen Syrakus noch nicht ausreichend befriedigt waren, führten sie beide eine tief in den Westen Siziliens reichende Kampagne, die allerdings abgesehen vom freundschaftlichen Gewinn von Segesta im Großen und Ganzen erfolglos war. ${ }^{12}$

\footnotetext{
9 Vgl. Polybius 1.11.3.

${ }^{10}$ Vgl. Eutrop 2.18.3 und 19.3; Silius Italicus 6.661 f.; Sueton, Tiberius 1. Vgl. Bleckmann (2002: 78-84).

11 Vgl. Plinius, NH 7.214; Censorinus, De die natali 23.7.

${ }^{12}$ Gemeinsames und getrenntes Vorgehen der Konsuln: Zonaras 8.9.16; Naevius frg. 3, „Manius Valerius consul partem exerciti in expeditionem duxit“. Einzelepisoden der Kampagne des Manius Valerius sind anscheinend auch auf dem Historiengemälde in der curia Hostilia dargestellt worden: zu Plinius, $n h$ 35.22, vgl. Molthagen (1979: 60-62); Bleckmann (2002: 88-90). Aus Polybius 1.16 ist dagegen nicht zu erkennen, dass Manius Valerius in dieser Kampagne Möglichkeiten nach einer getrennten Bewährung suchte. Sein Beiname Messala: Bleckmann (2002: 90 f.). Kampagne in den Westen Siziliens: vgl. hierzu Hoyos (1998: 108-110). Dass Heuss ( ${ }^{3}$ 1970: 47) für seine Darstellung des Abschlusses der Kampagne von 263 ausschließlich Polybios benutzt, veranlasst ihn zu problematischen Schlussfolgerungen über die angebliche Begrenztheit römischer Kriegsziele: vgl. Bleckmann (2002: 93, Anm. 2.).
} 


\section{Duilius und der neue Seekrieg}

Aristokratische Rivalität spielte auch im eskalierenden Seekrieg eine entscheidende Rolle, auch wenn ein Teil der Ereignisse durch die Gegenreaktionen Karthagos diktiert war, das sich überhaupt erst ab 262 in massiver Form engagierte, um die Positionen in Sizilien als Teil des eigenen Reichs zu halten. Diese Steigerung des karthagischen Engagements zwang die römische Politik, an der bereits im Westfeldzug von 263 vorskizzierten und für die Machtmittel Roms keineswegs besonders ehrgeizigen Besitznahme der Gesamtinsel festzuhalten und dabei auch den eigenen Einsatz zu vergrößern. Durch die Eroberung Agrigents, die nach einer langwierigen Belagerung erst dann möglich war, als die Karthager die Stadt verlassen hatten, zeigte sich viel deutlicher als in der insgesamt noch bescheidenen Kampagne von 263, dass die Beute aus den reichen griechischen Städten diese Vergrößerung des Einsatzes als lohnend erscheinen ließ. Um Karthago auf gleicher Augenhöhe zu begegnen, war der Bau einer Flotte unerlässlich. Gelang es, die Flotte der Karthager zu stellen, ergab sich für ehrgeizige Feldherrn die Möglichkeit, in der kurzen Zeit des eigenen Oberkommandos eine Entscheidung rasch zu erzielen.

Den Plan des Aufbaus einer Großflotte, der bereits einige Zeit in Rom diskutiert wurde und für den etwa der Konsular M'. Valerius im Senat eintrat, scheint der Konsul Gaius Duilius mit Erfolg in Senat und Volksversammlung durchgesetzt zu haben, nachdem die Winterpause von 261/260 dazu geführt hatte, dass ein großer Teil der in Sizilien errungenen römischen Positionen durch Aktionen der karthagischen Flotte wieder verloren gegangen war. Die straffe Organisation des in kürzester Zeit ausgeführten Flottenbaus und das Flottenkommando gaben dem Konsul zuvor nicht gekannte Machtbefugnisse in der Hand. Die Divergenzen in den Quellen lassen vermuten, dass die Frage des Kommandos der neuartigen Flotte mit heftigen Rivalitäten zwischen den beiden Konsuln verbunden war. Die Hauptquellen Polybios und Cassius Dio (Zonaras) sind sich lediglich darin einig, dass 260 nur einer der beiden Konsuln die prestigeträchtige und erfolgreiche provincia des Flottenkommandos erhalten hatte, während der andere Konsul den weniger spektakulären Landkrieg führen sollte. Sie bieten aber, was die Rolle des C. Duilius und des Cn. Cornelius Scipio betrifft, unvereinbare Angaben. Nach Polybios war es Cn. Cornelius Scipio, „der von den Römern das Kommando über die Seestreitkräfte“ erhalten hatte. Erst nachdem sein Kollege C. Duilius vom „Mißgeschick des Befehlshabers der Flotte erfuhr“ - Scipio war bei Lipara gefangen genommen worden, was ihm in der römischen Tradition zum Beinamen Asina verhalf -, habe dieser „die Legionen des Landheeres den Kriegstribunen übergeben“ und sei „,selbst zur Flotte hinübergefahren““ ${ }^{13}$ Bei Cassius Dio (Zonaras) hat dagegen Duilius von Anfang an das Kommando über die große neu aufzubauende römische Flotte inne und leitet von Rom aus die Flottenrüstung. Scipio, der nur das Kommando über die Landtruppen erhalten hat, ist mit dieser Verteilung der Aufgabenbereiche (provinciae) unzufrieden und versucht, diese Benachteiligung auszugleichen, indem er mit der kleinen Flotte, die ihm für die Begleitung seiner Landoperationen zur Verfügung steht, einen Überraschungscoup gegen Lipara ausführt, der ihm zum Verhängnis wird. Für den gefangen genommenen $\mathrm{Cn}$. Cornelius wird nun der praetor urbanus als Kommandeur der Landtruppen ausgeschickt. Duilius folgt nach einer Weile mit der

${ }^{13}$ Vgl. Polybius 1.21.4 und 23.1. 
endlich fertiggestellten Hauptflotte, die er in Sizilien dann mit den berühmten „Raben“ ausstattet. $^{14}$

Gewöhnlich wird bei diesem Widerstreit der Versionen die Nachricht des Polybios vorgezogen. Da aber die Quelle, die Cassius Dio benutzt hat, offenkundig auf einen annalistischen Autor zurückgreift, der von der spätannalistisch-livianischen Tradition unabhängig ist und qualitätsvolle Informationen erhalten hat, ist nicht ausgeschlossen, dass diese Version das Richtige wiedergibt, zumal die Erzählung des Polybios darauf hin angelegt ist, das Verhalten des Scipio Asina im Sinne der scipionischen Familienlegende zu relativieren und die Meriten des Duilius zu schmälern. Sachlich lassen sich viele Gemeinsamkeiten zwischen der Erzählung des Dio-Zonaras und der Inschrift auf der Duilius-Säule feststellen, die als ein authentisches Dokument gelten darf und getreu widerspiegelt, welchen gewaltigen Prestigegewinn der aus einer plebejischen Aufsteigerfamilie stammende Duilius mit der ersten großen erfolgreichen römischen Seeschlacht erzielen konnte. ${ }^{15}$

Die Duilius-Säule wurde an prominenter Stelle im Forum Romanum (bei den Rostra) aufgestellt. Sie verdeutlichte vor allem die durch den großen Erfolg von Mylai errungene Popularität des Feldherrn. Denn es ist davon auszugehen, dass diese herausragende Ehrung durch Volksbeschluss (offenkundig auf Intervention der Volkstribunen) verliehen worden ist. Dabei gehörte die Aufstellung dieser Säule zu einem Ensemble von Maßnahmen und Ehrenbeschlüssen, die die Erinnerung am Sieg festhalten sollten. So erhielt Duilius nicht nur eine besondere Form des Triumphes, den triumphus navalis, sondern auch das exorbitante Privileg, sich in einer besonderen, offenkundig einen verkleinerten Triumph simulierenden Form nach Hause begleiten zu lassen. Auch der Votivtempel, den Duilius nach seinem großen Erfolg errichten ließ, sollte in mehrfacher Hinsicht an seine Großtaten als Sieger zur See erinnern. Er war dem Seegott Janus geweiht und stand nicht nur an der via triumphalis, sondern gleichzeitig in unmittelbarer Nähe der Docks, wo die Flotte gebaut worden war.

Einiges spricht dafür, dass dieser gewaltige Prestigegewinn von den Standesgenossen nicht neidlos akzeptiert wurde. Vielmehr hatte Duilius diesen Prestigegewinn in einem Klima erbitterter Rivalität mit den anderen Adligen erzielt, bei der das Volk als Schiedsrichter die Leistungen des Duilius hervorhob. Offenkundig haben deshalb Standesgenossen wie die Angehörigen Scipio Asinas alles darangesetzt, dass Duilius trotz seiner Erfahrungen und seines Prestiges im Seekrieg keine zweite Chance erhielt. Dafür könnte jedenfalls die weitere Karriere des Duilius sprechen, der sich zwar in unmittelbarem Anschluss an den Erfolg von Mylai noch die Zensur sichern konnte (258), der aber anschließend erst als hochbetagter Staatsmann 231 eine unbedeutende Diktatur zur Abhaltung der Wahlkomitien bekleiden konnte, während sein militärisch unfähiger Kollege, der hocharistokratische Scipio, bereits 254 ein neues Konsulat erhielt. ${ }^{16}$

\footnotetext{
${ }^{14} \mathrm{Vgl}$. Zonaras 8.10.8-9 und 11.1-5.

15 Zur Eroberung von Agrigent vgl. Polybius 1.18.8-19 und Zonaras 8.10.2-5 mit Bleckmann (2002: 98-100). Weiteres Engagement der Römer nach der Eroberung: Polybius 1.20.1-2. Die bewusste Formulierung des Kriegsziels (vollständige Vertreibung der Karthager aus Sizilien) bedeutete keinen Bruch mit der bisherigen Kriegführung in Sizilien, sondern führte nur den bisherigen Expansionskurs konsequenter und bewusster als zuvor fort. M'. Valerius und die Großflotte: FGrH 839 F 1,4; zur Datierung dieser Äußerung: Thiel (1954: 53). Nachricht des Polybius vorgezogen: Thiel (1954: 188).

${ }^{16}$ Duilius-Inschrift: ILLRP 319; zur Authentizität des Textes: Bleckmann (2002: 116-125); Kondratieff (2004: 10-14). Duilius' exorbitantes Privileg: besonders deutlich Florus 1.18.10; vgl. ferner Inscr. Ital. 13, 3, 13;
} 


\section{Imperiales Ausgreifen bis nach Afrika}

Der Seesieg von Mylai hatte zwar eine große Beute und das Prestige, eine alte Seemacht besiegt zu haben, eingebracht, bewirkte aber strategisch letztlich wenig. Nach dem Abzug des Duilius, der Ende Februar 259 in Rom triumphierte, nutzte Hamilkar die Winterpause, um seinerseits wieder rasch Terrain auf Sizilien gewinnen zu können. Deutlich wurde, wie ungeeignet die saisonale Beschränkung der römischen Kriegführung war, um einen gut organisierten, zu langfristiger strategischer Planung fähigen Staat wie Karthago in die Knie zu zwingen. Die römische Politik reagierte freilich nicht dahingehend, dass die Führungsstrukturen verbessert wurden und die langfristige Verfolgung militärischer Ziele möglich wurde. Vielmehr wurde das durch den Seesieg von Mylai erreichte Prestige des Duilius zum Vorbild der jährlich aufeinander folgenden Konsuln, die in ihrer Amtszeit ein Maximum an Erfolgen erreichen und hierzu in mitunter hochriskanten Aktionen die ihnen für wenige Wochen zur Verfügung stehende Flotte einsetzten. In wenig koordinierten, meist saisonalen, nur in seltenen Fällen durch die Verlängerung des Kommandos über die Winterpause hinweg geführter Kampagnen ging es zwar oft darum, zunächst Rückschläge auszugleichen, die in der Winterpause erlitten worden waren, wenn Karthago Terrain zurückzugewinnen versucht hatte. Aber in der Hauptsache wurde in immer weiter ausgreifenden Unternehmungen nach neuen und spektakulären Aktionsfeldern gesucht. Zu nennen sind vor allem die nur halb erfolgreiche, gleichwohl aber mit einem Triumph belohnte Expedition des L. Cornelius Scipio nach Korsika und Sardinien (259), die großflächigen, durch C. Atilius Calatinus durchgeführten Aktionen auf Sizilien, die mit einem erfolglosen Unternehmen gegen Lipara endeten, die Sardinienkampagne des C. Sulpicius Paterculus und schließlich der Überfall auf Malta durch die Flotte des C. Atilius Regulus, Konsul von 257 und Sieger der Seeschlacht von Tyndaris. Dabei zeigt eine genaue Betrachtung der Texte, dass die Afrikastrategie des Regulus keineswegs eine revolutionäre Wende in der Kriegführung war, sondern sich mit einer gewissen Folgerichtigkeit aus der Ausweitung zu immer kühneren Unternehmungen ergab. Diese Ausweitung hatte bereits 258 bei Sulpicius Paterculus zur Formulierung des Projekts geführt, von Sardinien nach Afrika zu fahren. ${ }^{17}$ Die Überfahrt nach Afrika war ein Ziel, das wohl auch hinter dem Überfall auf Malta im Jahre 257 stand. $^{18}$

Die Behauptung des Cassius Dio, die Konsuln des Jahres 256 seien gerade wegen ihrer Tüchtigkeit für die Afrikaexpedition gewählt worden, ${ }^{19}$ ist deshalb problematisch, weil M. Atilius Regulus, der Bruder des Konsuls von 257, erst nach dem Tode des zuerst gewählten Konsuls Caedicius als consul suffectus nachgewählt wurde. Möglicherweise trug aber der Einfluss des atilischen Familienclans dazu bei, dass nach der Wahl des Regulus nun alles auf eine Karte gesetzt wurde und beide Konsuln sich das Kommando einer in dieser Größe zuvor nicht gekannten Flotte teilten. Nachdem die Flotte bereits vor der Überfahrt nach Afrika vor der Südküste Siziliens bei Eknomos einen herausragenden Sieg errungen hatte, scheint es zunächst zu Friedensverhandlungen mit Karthago gekommen zu sein. Nach den

\footnotetext{
Pseudo-Aurelius Victor, De viris illustribus 38.4; Val. Max. 3.6.4; Livius per. 17; Silius Italicus 6.663-9. Votivtempel: Beck (2005: 226) mit Verweis auf Livius 40.51.6. Anders zur Beurteilung der späteren Karriere des Duilius allerdings mit durchaus erwägenswerten Argumenten: Beck (2005: 227).

${ }^{17} \mathrm{Vgl}$. Zonaras 8.12.4.

18 Vgl. Orosius 4.8.5; Naevius, frg. 37 Blänsdorf.

19 Vgl. Cassius Dio, frg. 43.20; Zonaras 8.12.8.
} 
Reparaturen und Nachrüstungen der Flotte in Messana wagten aber beide Konsuln den Kraftakt der Überfahrt nach Afrika. ${ }^{20}$

Diese Expedition verlief anfangs angesichts der Unterlegenheit der karthagischen Flotte völlig problemlos. Das Heer konnte in Afrika sicher landen und, nach der Eroberung von Aspis, das reiche Landgebiet Karthagos plündern und römische Kriegsgefangene befreien. Für die Winterpause setzte M. Regulus eine Verlängerung seines Kommandos durch, während Manlius Vulso nach Rom zurückkehrte und dort einen triumphus navalis feierte. In den Briefen, die Regulus in den Folgemonaten nach Rom schickte, ging es dem in Afrika verbliebenen Feldherrn darum, sich möglichst lange das Kommando und seine Prestigechancen zu sichern. Die Sorge vor dem Eintreffen des Nachfolgers motivierte ihn in der Hauptsache, nach weiteren großen Erfolgen während der Wintersaison mit den Karthagern Friedensverhandlungen aufzunehmen:

Marcus, der die Karthager zu Lande und zu Wasser geschlagen sah, jedoch in Sorge war, sein Nachfolger könne vorher aus Rom eintreffen und die Ehre des Sieges ernten, lud die Karthager zu Friedensverhandlungen ein. ${ }^{21}$

Diese Friedensverhandlungen waren jedoch dadurch erschwert, dass Regulus aufgrund der römischen Erwartungshaltungen und der großen römischen Opfer auf jeden Fall die Abtretung Siziliens und Sardiniens erreichen musste, weil sonst mit der Zustimmung des römischen Volkes nicht zu rechnen war. Nachdem die Verhandlungen gescheitert waren, kam es Regulus nunmehr darauf an, um jeden Preis die Entscheidung in seiner Amtszeit zu erzwingen. Aus diesem Grund nahm er eine Schlacht in der Ebene bei Tunis an, und zwar unter ungünstigen Bedingungen und ohne sich mit den neuen taktischen Errungenschaften vertraut zu machen, die die karthagische Truppe dem gerade eingetroffenen Militärexperten Xanthippos zu verdanken hatte. Der nobilitätstypische Wunsch, ,unter den eigenen Auspizien die größten Taten auszuführen“"22, erklärt damit sowohl den Erfolg des Atiliers, der das schon zuvor von anderen angestrebte Afrikaprojekt durch bisher noch nie aufgebrachte Flottenrüstungen durchsetzen konnte, als auch sein Scheitern.

$\mathrm{Zu}$ einer unmittelbaren Korrektur der Kriegführung kam es nach dem Scheitern der Friedensbemühungen des Regulus keineswegs. Vielmehr war die mit immerhin 350 Schiffen unternommene Expedition des Servius Fulvius Paetinus und des M. Aemilius Paullus, der Konsuln des Jahres 255, keineswegs nur ein bloßes Nachspiel der Regulus-Expedition, sondern neben der Rettung der in Afrika verbliebenen römischen Bürger verfolgten die beiden Konsuln ehrgeizigere Ziele. Nachdem Kossyra geplündert und eine karthagische Flotte beim Kap Hermaion vernichtet worden war, versuchten die beiden Konsuln, sich in Afrika festzusetzen, mussten dann aber aufgrund von Verpflegungsschwierigkeiten zurückkehren. Beim Versuch, diese Erfolgsbilanz auf der Rückfahrt durch Machtdemonstrationen gegenüber sizilischen Griechen abzurunden, fuhren die Konsuln gegen den Rat der nautischen Experten an der südsizilischen Küste entlang, wo sie auf der Höhe von Kamarina in einen schweren Sturm gerieten, der die Flotte fast völlig zerstörte. Trotz die-

\footnotetext{
${ }^{20}$ Zur Kampagne von 257, die nach der Schlacht von Tyndaris fortgeführt wurde: Bleckmann (2002: 157 f.). Zum bei Zonaras 8.15.8 richtig wiedergegebenen Verwandtschaftsverhältnis der Reguli: Beck (2005: 231, Anm. 11).

21 Polybius 1.31.4.

${ }^{22}$ Plinius, NH $7.139 \mathrm{f}$.
} 
ses Scheiterns konnten beide Konsuln sich einen Triumph sichern, und Aemilius Paullus wurde zusätzlich in gleicher Form wie Duilius durch eine columna rostrata geehrt.

Die Konsuln des Jahres 254 verzettelten sich zu lange in einzelnen Operationen an der Nordküste - wo ihnen insbesondere die Eroberung Palermos gelang -, um mit ihrer großen Flotte weiter nach Afrika zu gelangen. Eine grundsätzliche strategische Wende war aber mit der Kampagne von 254 nicht eingeschlagen worden. Denn im Jahre 253 fuhr die römische Flotte über die jetzt besser gesicherte nordsizilische Küste erneut nach Afrika, konnte sich aber wegen des erbitterten karthagischen Widerstands nirgends festsetzen und musste schließlich, nachdem sie bis zur Kleinen Syrte gelangt war, wieder zurückkehren. Bei der überstürzten Rückfahrt von Palermo nach Italien wurden erneut 150 Schiffe durch den Sturm vernichtet. Das bedeutete bei einer Bemannung mit 200 oder sogar 300 Ruderern einen Verlust von mindestens 30.000 Römern und Bundesgenossen.

\section{Die Einstellung des Seekriegs und der Prozess gegen P. Claudius Pulcher}

Die Geschichte der großen und verlustreichen Flottenfahrten der 250er Jahre wurde weder durch einen Wechsel in der Strategie, den die aus der Nobilität rekrutierten Generäle vornahmen, beendet noch dadurch, dass der Senat, dessen Führungskompetenzen für diese Zeit nur undeutlich abgrenzbar sind, sich vom Seekrieg auf den Landkrieg verlegte. Entscheidend war vielmehr, dass angesichts der großen menschlichen Verluste, aber auch der durch die Flottenrüstung gegebenen materiellen Belastung der Widerstand im Volk gegen die Rekrutierungen für den Flottendienst immer mehr zunahm. Die anfängliche Interessenkoalition zwischen Nobilität und Volk, die darauf beruht hatte, dass für beide Parteien der Seekrieg lukrativ war, und die etwa in der Begeisterung für die Erfolge des Duilius ihren Niederschlag gefunden hatte, war durch die Wiederholung der Katastrophen zerbrochen. Dass das Verbot weiterer Seekriegführung (vermutlich nach der Agitation durch einige Volkstribunen) unter dem Eindruck der Katastrophe der Flottenfahrt von 253 von der Volksversammlung beschlossen wurde, wird bei Polybios, der nur von den „Römern“ als handelndes Kollektiv berichtet, nicht so deutlich zur Sprache gebracht wie bei DioZonaras, dessen Version hier gegenüber derjenigen der livianischen Tradition eindeutig den Vorzug verdient:

Als sie [die Konsuln; d. Verf.] nach Hause zurückkehrten, hatten sie es mit einem Sturm zu tun, und ihre Flotte wurde zerstört. Daher glaubte das Volk, wegen der mangelnden Erfahrung im Seewesen geschädigt zu werden, und faßte durch Abstimmung den Beschluß, vom übrigen Meer [das heißt den außerhalb der Küste Italiens gelegenen Gewässern; d. Verf.] sich fernzuhalten und nur mit einigen Schiffen Italien zu bewachen. ${ }^{23}$

\footnotetext{
${ }^{23}$ Aemilius Paullus' columna rostrata: Sehlmeyer (1999: 119f.). Der Bau der Flotten bis 253 kann nur durch Umlageverfahren finanziert worden sein, die die politisch besonders einflussreiche erste Klasse besonders belasteten; vgl. zur materiellen Belastung Appian, Sik. 1.1. Das spätere private Engagement von ersten Bürgern des Staates (Pol. 1.59) kopiert nur das anfangs staatlich erzwungene Verfahren. Zur erfolglosen Agitation des Nautius gegen die Flottenfahrt des Regulus vgl. Bleckmann (2002: 180, Anm. 2). „Als sie nach Hause zurückkehrten“: Zonaras 8.14.6. In der livianischen Tradition trifft der Senat die Entscheidung (Eutrop 2.23; Orosius 4.9.12). Zur Bevorzugung dieser Tradition durch Rotondi (1912), Elster (2003) und so weiter vgl. die Argumentation bei Bleckmann (2002: 179, Anm. 2).
} 
Dass dieses Gesetz zunächst nur wenige Jahre Gültigkeit behielt, lag an dem herausragenden Sieg, den Metellus als Prokonsul mit seinen Landtruppen in der Winterpause von 251/250 über die Karthager bei Panormos erringen konnte und für den er in exzessiver, das spätere Selbstbewusstsein der Meteller prägender Weise geehrt wurde. Was den Konsuln der vorangegangenen Jahre nicht gelungen war, wollten die Konsuln des Jahres 250 nun endlich durchsetzen, indem sie durch eine Fahrt nach Afrika den Krieg beendeten. In der durch den Sieg von Panormos hervorgerufenen Euphorie konnten sie erfolgreich in der Volksversammlung eine Entscheidung durchsetzen, die implizit den Beschluss von 252 aufhob und ihnen ausdrücklich die Flottenfahrt nach Afrika als Auftrag zuwies. Der Angriff, den diese auf 200 Schiffe vergrößerte Flotte gegen den karthagischen Stützpunkt Lilybaeum vornahm, sollte nicht die Eroberung Siziliens vollenden; vielmehr planten die ehrgeizigen Konsuln C. Atilius Regulus und L. Manlius Vulso, die bereits 257 beziehungsweise 256 amtiert und große Flottenverbände geführt hatten, die Einnahme als Auftakt für die Überfahrt nach Karthago. Allerdings leistete nicht nur die karthagische Besatzung von Lilybaeum unerwartet zähen Widerstand, sondern es gelang auch noch einem großen karthagischen Entsatzkorps unter der Leitung Hannibals, in die Stadt zu gelangen, so dass sich das Großunternehmen hinzog und die römische Streitmacht, von der nur ein Teil über die Winterpause die Belagerung der Stadt fortführte, in ernste Schwierigkeiten geriet. Zusätzlich nutzten die Karthager die Lähmung der römischen Kriegführung aus, um nun ihrerseits mit ihrer Flotte Angriffe bis nach Italien zu wagen. ${ }^{24}$

Vor dem Hintergrund dieser kritischen Situation müssen die Aktionen des P. Claudius Pulcher, des Konsuls von 249, beschrieben werden, dessen Scheitern zu der schwersten innenpolitischen Krise im langen Krieg, aber auch zur Erneuerung des gesetzlichen Verbots der Seekriegführung führte. In der ursprünglichen Verteilung der provinciae hatte Iunius Pullus die Flotte erhalten, mit der er entlang der Südküste Siziliens nach Lilybaeum fahren sollte. Claudius hatte dagegen das Kommando über Landtruppen, mit denen er der Nordküste entlang marschierte, um die Belagerungstruppen bei Lilybaeum zu unterstützen. Allerdings gab er sich mit dieser eher beschränkten militärischen Aufgabe nicht zufrieden, sondern ließ in Lilybaeum eine neue große Flotte bauen und die als Schiffsbesatzungen ausgewählten Truppen teils unter brutalen Methoden für den Seekampf trainieren. Der Versuch, die karthagische Flotte beim zweiten wichtigen karthagischen Stützpunkt, bei Drepana, zu überraschen, misslang freilich völlig. Claudius war völlig überrascht, als er erkennen musste, ,daß die Feinde wider Erwarten weder flohen noch auf seinen Angriff panisch reagierten“ ${ }^{25}$. Es gelang nicht, seine teilweise in den Hafen von Drepana eingefahrene Flotte zu ordnen. Ein großer Teil der chaotisch manövrierenden Schiffe wurde versenkt, ein anderer von den Karthagern erbeutet.

Das taktische Versagen des Claudius wäre ihm allein - hier war das römische Volk gegenüber den aus der Nobilität rekrutierten Generälen immer noch überraschend nachsichtig - nicht zum Verhängnis geworden, wohl aber die Art und Weise, in der sich der nach Rom zurückgekehrte Claudius Pulcher verhielt, nachdem auch die Flotte des Iunius

\footnotetext{
${ }^{24}$ Flottenfahrt nach Afrika 250 durch Volksversammlung zugewiesen: Zonaras 8.15.3, „,Sie [die Römer; d. Verf.] beschlossen durch Abstimmung, dass die Konsuln nach Afrika fahren sollten." Karthagische Angriffe bis nach Italien: Polybius 1.41.4. Zu den oft problematischen Interpretationen in der Sekundärliteratur: Bleckmann (2002: 183).

${ }^{25}$ Polybius 1.50.1.
} 
Pullus durch einen Seesturm vernichtet worden war und es im Senat zu erbitterten und anscheinend sehr handgreiflichen Diskussionen über die Fortführung des Krieges kam. Eine gegen Claudius eingenommene Adelsfraktion entriss dem Konsul noch während seiner Amtszeit das Oberkommando, indem es ihm die Ernennung eines Diktators befahl. Claudius konterte, indem er demonstrativ seinen Klienten Claudius Glicia zum Diktator erhob, der de facto von seinen Weisungen abhing. Dieses Verhalten ist in einer Konfliktsituation verständlich, in der das genaue Kräfteverhältnis von Magistrat und dem (durch die lex Ovinia in seiner Rekrutierung und in seiner Stellung gegenüber dem Magistrat fundamental veränderten) Senat noch nicht völlig geklärt war und in der selbstbewusste Konsuln wie Claudius sich nicht als Exekutive des Senats und der Nobilität verstanden. Erst in einer zweiten Stufe der Auseinandersetzung lenkte Claudius schließlich ein und erhob statt des zur Abdankung gezwungenen Glicia den bereits bewährten Konsular A. Atilius Calatinus zum Diktator.

Die rücksichtslose Amtsführung des Claudius führte dazu, dass ihm nach seinem Amtsjahr der Prozess gemacht wurde. Im Unterschied zur radikalen griechischen Demokratie waren der römischen Republik der Generalsprozess und die Vorstellung, die Vernachlässigung der Interessen des Volkes ahnden zu müssen, an sich fremd. In der psychologisch aufgeladenen Atmosphäre, die nach den Niederlagen und Katastrophen von 249 geherrscht haben muss und die auch im Kult ihren Niederschlag fand, sahen aber einige Volkstribunen eine günstige Gelegenheit zur Agitation und zur politischen Profilierung, indem sie einen Prozess gegen Claudius initiierten. Die Angaben der historiografischen Quellen, die teilweise von einer erst in der Späten Republik ausgeschmückten negativen Legende über die angebliche Arroganz der Claudier gezeichnet sind, lassen allerdings nicht genau erkennen, was und in welcher Form bei diesem Prozess verhandelt wurde und in welcher Weise er von damaligen innenpolitischen Grundproblemen bestimmt war. Schon das inkriminierte Delikt ist unklar. Gewöhnlich wird angenommen, Claudius Pulcher sei sein frevelhafter Umgang mit den religiösen Riten unmittelbar vor der Schlacht vorgeworfen worden. ${ }^{26}$

Nach der Deutung von K.-J. Hölkeskamp dagegen sei der eigentliche Prozessgrund die normwidrige Erhebung des Glicia und die Herauslösung des Claudius aus dem aristokratischen comment gewesen, während die sakralrechtlichen Verstöße nur einen Vorwand abgegeben hätten. Eine genaue Lektüre der einschlägigen Passagen zu den angeblichen Verstößen zeigt allerdings, dass dort Bezüge zum Prozess nur indirekt hergestellt werden: Der frevelhafte Umgang mit den Auguralriten führte zur Niederlage, wegen der wiederum dem Konsular der Prozess gemacht wurde. ${ }^{27}$ Vermutlich waren also die sakralrechtlichen Verstöße nicht einmal auf einer formalen Ebene Gegenstand des Prozesses, sondern die Art der Kriegführung selbst, durch die das römische Volk schwer geschädigt worden war. Anklagegrund war in diesem Kapitalverfahren, für das die Volkstribunen auf die Amtshilfe von Magistraten cum imperio angewiesen waren, wohl der recht vage Tatbestand des „Hochverrats“, der perduellio, die mit dem aus der athenischen Geschichte bekannten Delikt der prodosia, dem Verrat von Volksinteressen, vergleichbar ist. Das wird auch in der

\footnotetext{
${ }^{26}$ Lex Ovinia und Senat: Cornell (2000); Bleckmann (2002: 142). psychologisch aufgeladene Atmosphäre: vgl. zur Einrichtung der ersten Saecularspiele in diesem Zeithorizont die allerdings insgesamt sehr unklaren Angaben bei Zosimus 2.4.1 (wegen der Seuchen und Kriege: für die „Seuchen“ ist auf die Epidemie im Winterlager von Lilybaeum 250/249 zu verweisen, vgl. Zonaras 8.15.2); Censorinus 17.10.

${ }^{27}$ Hölkeskamp (1990); vgl. Cicero, De nat. deor. 2.7; Schol. Bob. p. 90 Stangl; vgl. Bleckmann (2002: 196).
} 
ältesten Quelle zum Prozess gegen Claudius, nämlich bei Polybios (1.52.3), deutlich, bei dem der Auspizienfrevel keine Rolle spielt:

Publius aber wurde von den Römern verachtet und war heftig beschuldigt worden, daß er die Geschäfte aufs Geratewohl und ohne Verstand anging und daß er durch sein Verschulden Rom schwere Verluste zugefügt hatte. Deshalb wurde ihm auch später der Prozess gemacht, wo er schweren Strafen und Gefahren ausgesetzt wurde.

Die Vorstellung, Magistrate müssten sich wegen Fehlhandlungen verantworten, ist zwar in Rom niemals über rudimentäre Ansätze hinausgekommen. Gleichwohl ist der Prozess kein Einzelfall, sondern hat Parallelen bis zum Prozess gegen Servilius Caepio nach der Katastrophe von Arausio in Gallien (105 vor Christus). Entscheidend für das Zustandekommen solcher, dem Amtsverständnis selbstherrlicher Nobiles völlig widersprechender Prozesse war eine Konstellation, in der agitierende Volkstribunen in der Nobilität Verbündete finden konnten.

Angesichts des Fehlens einer zeitnahen Überlieferung zum Prozessverlauf ist schwer festzustellen, ob im Prozess gegen Claudius die gesamte Nobilität auf Seiten der Volkstribunen und in geschlossener Frontstellung gegen den Einzelgänger Claudius stand und ob der Prozess einen Beleg für den so genannten Funktionswandel des Volkstribunats in der Klassischen Republik bietet, durch den die Volkstribunen nicht mehr als Vertreter plebejischer Sonderinteressen, sondern ausschließlich als Instrumente der Senatspolitik agierten. Folgt man den späten Quellen, die allein überhaupt eine Darstellung der Prozessdetails bieten, gab es jedenfalls eine eindrucksvoll geschlossene Frontstellung von Volkstribunen und Nobilität gegen Claudius vermutlich nicht. Denn der zunächst von den Volkstribunen mit der Amtshilfe von Magistraten aus der Nobilität durchgeführte Kapitalprozess vor den Kuriatkomitien konnte deshalb nicht zu Ende geführt werden, weil andere Amtsträger, die zu Claudius hielten, diesen Prozess erfolgreich durch die üblichen Mittel der Obstruktion wie der erfolgreichen Beobachtung bedenklicher Vorzeichen behindern konnten. ${ }^{28}$ Gegen eine Wiederaufnahme des Kapitalprozesses legten offenkundig auf der Seite des Claudius stehende Volkstribunen Widerspruch ein, ${ }^{29}$ auch wenn sie sich sonst in der Verteidigung des unbeliebten Konsulars nicht weiter hervorwagten. Wegen dieses aus der Nobilität vorgebrachten Widerstandes mussten die für die Betreibung des Prozesses verantwortlichen Volkstribunen Pullius und Fundanius auf die Durchführung des Kapitalverfahrens vor der Volksversammlung verzichten und ein Multverfahren vor dem concilium plebis durchführen, das schließlich mit einer Geldstrafe für Claudius endete.

Bei genauer Betrachtung entspricht der Claudius-Prozess damit dem auch aus der Späten Republik bekannten Bild römischer Innenpolitik: Von den Volkstribunen waren nur einige, aber keineswegs alle, bereit, sich für die Belange der Nobilität einspannen zu lassen, während umgekehrt auch die Nobilität gespalten war und einige die Agitation von im „Volksinteresse“ tätigen Tribunen unterstützen, andere diese aber bekämpften. Deutlich ist auch, dass sich für Fundanius Fundulus, durch den der Prozess maßgeblich betrieben wurde, die populare Agitation in seiner späteren Karriere bezahlt machte, indem er - der cursus honorum war um diese Zeit noch nicht festgelegt - 246 Ädil $^{30}$ und 243 Konsul

\footnotetext{
${ }^{28}$ Vgl. Schol. Bob. p. 90 Stangl.

${ }^{29}$ Vgl. Schol. Bob. p. 90 Stangl.

${ }^{30}$ In dieser Zeit profilierte er sich wieder als Vertreter der Volksinteressen durch einen Prozess gegen die Schwester des Claudius: Valerius Maximus 8.1, damn. 4; Gell. 10.6; Sueton, Tiberius 2.3.
} 
wurde. Nur eine auf wenige Personen der römischen Geschichte zentrierte Legendenbildung hat dazu beigetragen, dass Fundanius Fundulus als einer der großen populares des dritten Jahrhunderts vergessen worden ist und nur noch wenige „Vorfahren“ der Gracchen wie Flaminius in Erinnerung geblieben sind.

Der Prozess gegen Claudius war dabei nur ein Teil der Agitation, die sich gegen die Fortsetzung des verlustreichen Seekrieges richtete. In der gleichen Zeit - nach Zonaras im Jahre 247 - wurde das bereits 252 beschlossene gesetzliche Verbot der Seekriegführung ,wegen der Unglücksfälle und wegen der materiellen Aufwendungen“ durch Plebiszit erneut eingeschärft. Für die Rückkehr zum Landkrieg in den folgenden Jahren war damit nicht die strategische Neuorientierung der einsichtig gewordenen Nobilität verantwortlich, sondern eine ,populare“ Gesetzgebungsinitiative, die in Zukunft die Plebs vor hohen materiellen und menschlichen Verlusten durch die leichtsinnige Seekriegführung einiger Vertreter der aristokratischen Elite schützen sollte. Diesen Vorgaben entsprechend konnte der Krieg in den folgenden Jahren nur als eine Serie eher kleinteiliger Landoperationen durchgeführt werden, in denen trotz einiger Territorialgewinne der von Fundanius Fundulus 243 in Aussicht gestellte Durchbruch nicht gelang. Immerhin konnten die vom langwierigen Krieg geschwächten Karthager neben einigen Festungen im Westen Siziliens vor allem den Besitz der getreidereichen und für das karthagische Imperium besonders wichtigen Insel Sardinien für sich behaupten. ${ }^{31}$

\section{Lutatius Catulus und das Ende des Ersten Punischen Kriegs}

Nach einigen Jahren eines nur mäßig erfolgreichen Landkrieges gegen die Karthago noch verbliebenen und auf dem Seeweg versorgten Positionen konnte die Rückkehr zum Seekrieg nur deshalb erfolgen, weil formal die für 242 von den beiden Konsuln ausgerüstete Flotte nur ein Freiwilligenaufgebot war. ${ }^{32}$ Dabei machte man sich die Tatsachen zunutze, dass in dieser Zeit ein Monopol des römischen Staates auf die Durchführung kriegerischer Aktionen zumindest für den Seekrieg nicht existierte und dass unternehmungslustige Privatleute in den Jahren nach 247 einen lukrativen Kaper- und Piratenkrieg gegen Karthago in oft ziemlich großen Unternehmungen fortgeführt hatten. ${ }^{33}$ Auf diese Weise machte es keine Schwierigkeit, trotz der längeren Pause im Flottenkrieg eine neue Flotte auszurüsten. Treibende Kraft war C. Lutatius Catulus, dem es zwar gelang, seinen Kollegen durch die Aktivierung religiöser Bedenken auszubooten, der sich aber dann das Flottenkommando mit dem selbstbewusst seinen Anteil am Ruhm reklamierenden Prätor Valerius Falto teilen musste.

Die entscheidende Aktion des Lutatius Catulus gelang ihm erst, als die Zeit seines Konsulats bereits abgelaufen und seine Amtszeit bereits verlängert worden war, vielleicht auf Betreiben seines Bruders und Amtsnachfolgers Q. Lutatius Cerco. Dabei scheint er - wie viele seiner Amtsvorgänger auch - extrem risikofreudig vorgegangen zu sein, um die karthagische Versorgungsflotte, die in Richtung auf Drepana unterwegs war, abzufangen. Aus

\footnotetext{
${ }^{31}$ Seekriegsverbot erneuert: Zonaras 8.16.2. Indirekt durch Polybios 1.59.1 bestätigt. Vgl. gegen Walbank (1957: 125) Bleckmann (2002: 192, Anm. 5). Siegesgewissheit des Fundanius Fundulus: Diodor 24.9.2, vgl. Polybius 1.59.1.

${ }^{32}$ Vgl. Polybius 1.59.6.

${ }^{33} \mathrm{Vgl}$. Zonaras 8.16.8.
} 
den Quellen geht nämlich hervor, dass der Konsul trotz des stürmischen Wetters den Befehl zur Ausfahrt gab. Das riskante Unternehmen ging aber gut aus, auch deshalb, weil die beladenen karthagischen Schiffe weniger manövrierfähig waren als die der Römer. Der Sieg bei den Aegatischen Inseln (241 vor Christus) endete gleichwohl nicht mit der völligen Vernichtung der karthagischen Flotte, und sicher ist darüber hinaus, dass auch von römischer Seite Einbußen hingenommen werden mussten. Vom ursprünglich wesentlich ehrgeizigeren, in der Tradition früherer Unternehmungen formulierten Ziel, Karthago selbst anzugreifen, war man noch weit entfernt. ${ }^{34}$

Über die Motive, die Lutatius Catulus veranlassten, nach seinem Seesieg gleichwohl den Friedensschluss mit Karthago zu betreiben, schreibt Dio-Zonaras:

Die Karthager schickten dem Catulus Gesandte, um über den Frieden zu verhandeln. Dieser war geneigt, den Krieg zu beenden, weil seine Amtszeit gerade auslief, weil er ferner nicht glaubte, in kurzer Zeit Karthago zerstören zu können, und weil er nicht seinen Nachfolgern den durch seine eigenen Mühen gewonnen Ruhm überlassen wollte. ${ }^{35}$

Es war also, wenn man Dio-Zonaras folgt, nicht die völlige Erschöpfung, wie sie von Polybios (1.62.7) in den Vordergrund gestellt wird, die Lutatius friedensbereit machte, sondern die Erkenntnis, dass während der kurzfristigen Verlängerung seines Kommandos die Zeit für eine Fahrt nach Afrika nicht mehr reichen würde und dass es für das eigene Prestige besser war, einen den Krieg beendenden Friedensvertrag durchzusetzen statt einem Nachfolger den Ruhm der völligen Unterwerfung Karthagos zu überlassen. Aus diesem Grund wurden Karthago Friedensbedingungen diktiert, die durchaus moderat waren und insbesondere die wichtige Insel Sardinien bei Karthago beließen.

Gegen diesen für Karthago zu günstigen Vertragsentwurf gab es in Rom heftige Proteste, die insbesondere von denen formuliert wurden, deren Ehrgeiz auf die Fortführung des Krieges und den Angriff in Afrika angewiesen war und die in der Volksversammlung die Ablehnung des Vertragsentwurfs durchsetzen konnten. ${ }^{36}$ Dass der Krieg dann doch nicht weiter geführt wurde, war einzig dem für Lutatius Catulus glücklichen Umstand zu verdanken, dass sein Nachfolger im Konsulat der eigene Bruder Lutatius Cerco war, der durch einige geschickte Modifikationen des Vertragsentwurfs dann schließlich die Ratifikation des Friedens durchsetzen konnte. Weil der Frieden von 241 nur mit äußerst knapper Mehrheit und in einem Klima heftiger innenpolitischer Auseinandersetzungen durchgesetzt worden war, ist es nicht verwunderlich, dass er nur wenige Jahre später durch die zusätzlich erzwungene und für Karthago besonders bittere Abtretung Sardiniens ,korrigiert" wurde, als Karthago durch den Söldneraufstand wehrlos geworden war.

\section{Bilanz}

Eine genaue Betrachtung der Wechselfälle des Ersten Punischen Krieges zeigt deutlich, dass dieser Krieg von römischer Seite zwar einerseits ohne einen gewissen Grundkonsens zwischen den Mitgliedern der Nobilität nicht durchführbar gewesen wäre, dass aber

\footnotetext{
${ }^{34} \mathrm{Zu}$ Lutatius Catulus und Lutatius Cerco sowie zum ursprünglichen Projekt der Afrikafahrt: Bleckmann (2002: 214-218) mit Verweis auf Valerius Maximus 1.1.2, gegen Thiel (1954: 320).

35 Zonaras 8.17.3.

${ }^{36}$ Explizit zu den Motiven der Ablehnung Zonaras 8.17.5 f. Das Faktum der Ablehnung bei Polybius 1.63.8, der im Rückblick verfassungsrechtliche Gründe anführt; vgl. Polybius 3.21.2 und Livius 21.18.10.
} 
andererseits konkrete Entscheidungen in einer durchaus gespannten und konfliktreichen innenpolitischen Atmosphäre fielen und dass das sallustische Bild einer harmonischen Einigkeit im Kampf gegen den äußeren Feind für diese Phase der Republikgeschichte in seiner Einseitigkeit nicht zutreffen kann. Die relativ große Bedeutung der Volksversammlung in dieser Zeit, die 264 Appius Claudius gegen die Bedenken des Senats mit einem Sonderkommando ausstattete oder 241 den ersten Friedensvertrag nicht ratifizierte, erklärt sich nicht daraus, dass Rom in dieser Zeit eine Demokratie war, sondern daraus, dass im inneraristokratischen Wettstreit der Entscheidung und Schiedsrichterrolle der Volksversammlung eine besonders große Bedeutung zugewiesen wurde und dass durch die Einbindung in den Seekrieg breitere Kreise von den politischen Entscheidungen betroffen waren. ${ }^{37}$

Die Instrumentalisierung der Volksversammlung bis hin zu demagogischen, mit der späteren Epoche der Gracchen durchaus vergleichbaren Formen ist nur ein Teilaspekt eines heftigen inneraristokratischen Wettstreits, der gerade auch die Amtsführung vieler sich unsolidarisch verhaltender Magistrate prägte, wenn man provincia-Zuweisungen ignorierte oder bei strategischen Entscheidungen darauf achtete, dem Amtsnachfolger keinen Anteil am erworbenen Ruhm zu überlassen. Der Konkurrenzkampf um Möglichkeiten des Erwerbs von militärischem Ruhm erklärt einerseits einen Großteil der imperialen Dynamik der Mittleren Republik, wurde aber andererseits bisweilen in Formen geführt, die das Wohl des Gesamtstaates gefährdeten. Gewiss fehlen in dieser Zeit des inneraristokratischen Konkurrenzkampfes die extremsten, aus der Späten Republik vertrauten Erscheinungen wie die Privatarmeen und Heeresklientelen, die politischen Morde und die Versuche, persönliche Macht gegen den mos maiorum institutionell langfristig abzusichern. Aber in den kaum gezügelten politischen Ambitionen einzelner Vertreter der Führungsschicht des dritten Jahrhunderts zeichnen sich Verhaltensmuster ab, die mit denen der Aristokraten der späteren Republik durchaus vergleichbar sind. Die viel gebrauchte Rede von der „Desintegration“ der Nobilität ist daher ungeeignet, um die Entwicklungsgeschichte der Nobilität zu bezeichnen. ,Desintegration“ suggeriert, dass eine anfangs harmonisch miteinander lebende Führungsschicht immer deutlicher zerfällt, während in Wirklichkeit ein erbitterter Konkurrenzkampf, der immer wieder die Grenzen der Standessolidarität belastete, von Anfang an vorhanden war. Dieser Konkurrenzkampf wurde aber zum Schluss aufgrund der Größe des Imperiums mit Machtmitteln ausgetragen, die die Grenzen der ohnehin immer nur schwachen gesamtstaatlichen Institutionen der Republik sprengten.

\section{Literatur}

BECK, Hans (2005). Karriere und Hierarchie. Die römische Aristokratie und die Anfänge des cursus honorum in der mittleren Republik. Berlin.

Bleckmann, Bruno (1998). „Regulus bei Naevius: Zu Frg. 50 und 51, Blänsdorf“, Philologus 142(1), 61-70.

Bleckmann, Bruno (1999). „Rom und die Kampaner von Rhegion“, Chiron 29, 123-146.

Bleckmann, Bruno (2002). Die römische Nobilität im Ersten Punischen Krieg. Untersuchungen zur aristokratischen Konkurrenz in der Republik. Berlin.

BleICKen, Jochen (1975). Lex Publica. Gesetz und Recht in der römischen Republik. Berlin.

CÀssola, Filippo (1962). I gruppi politici Romani nel III secolo A.C. Triest.

37 Vgl. Millar (1989). Zu dieser Debatte vgl. Hölkeskamp (2006: 363 mit Anm. 9). 
Cornell, Timothy J. (2000). „The Lex Ovinia and the Emancipation of the Senate“, in: Christer BruUn (Hrsg.). The Roman Middle Republic. Politics, Religion and Historiography. Rom, 6990.

Elster, Marianne (2003). Die Gesetze der mittleren römischen Republik. Text und Kommentar. Darmstadt.

Heuss, Alfred $\left({ }^{3} 1970\right)$. Der erste Punische Krieg und das Problem des römischen Imperialismus. Zur politischen Beurteilung des Krieges. Darmstadt. (1. Auflage 1949)

HöLKESKAMP, Karl-Joachim (1987). Die Entstehung der Nobilität. Studien zur sozialen und politischen Geschichte der römischen Republik im 4. Jhdt. v. Chr. Stuttgart.

HÖLKESKAMP, Karl-Joachim (1990)., „Senat und Volkstribunat im frühen 3. Jhdt. v. Chr.“, in: Walter EDER (Hrsg.). Staat und Staatlichkeit in der frühen Republik, Akten eines Symposiums 12.-15. Juli 1988. Stuttgart, 437-457.

HöLKESKAMP, Karl-Joachim (2001). ,Fact(ions) or Fiction? Friedrich Münzer and the Aristocracy of the Roman Republic - then and now", International Journal of the Classical Tradition 8, 92-105.

HölKeskamp, Karl-Joachim (2004). Senatus Populusque Romanus. Die politische Kultur der Republik - Dimensionen und Deutungen. Stuttgart.

HÖLKESKAMP, Karl-Joachim (2006). „Konsens und Konkurrenz. Die politische Kultur der römischen Republik in neuer Sicht“", Klio 8, 360-396.

Hoyos, B. Dexter (1998). Unplanned Wars. The Origins of the First and Second Punic Wars. Berlin und New York.

KondratiEFF, Eric John (2004). „The Column and Coinage of C. Duilius: Innovations in Iconography in Large and Small Media in the Middle Republic", Scripta Classica Israelica 23, 1-39.

LIPPOLD, Adolf (1963). Untersuchungen zur Geschichte des römischen Konsulates von 264 bis 201 v. Chr. Bonn.

Millar, Fergus (1989). „Political Power in Mid-Republican Rome: Curia or Comitium?“, Journal of Roman Studies 79, 1-11.

Molthagen, Joachim (1979). „Der Triumph des M’. Valerius Messala und die Anfänge des Ersten Punischen Krieges“, Chiron 9, 53-72.

MÜNZER, Friedrich (1920). Römische Adelsparteien und Adelsfamilien. Stuttgart.

Rotondi, Giovanni (1912). Leges Publicae populi Romani. Mailand.

SeHLMEYER, Markus (1999). Stadtrömische Ehrenstatuen der republikanischen Zeit. Stuttgart.

Staveley, E. Stuart (1989). „Rome and Italy in the Early Third Century“, in: The Cambridge Ancient History. Second Edition VII,2: The Rise of Rome to 220 B.C. Cambridge, 420-455.

THIEL, Johannes Hendrik A. (1954). A History of Roman Sea-Power before the Second Punic War. Amsterdam.

Walbank, Frank William (1957).A Historical Commentary to Polybius I. Oxford. 
